Editorial

\title{
Designing for Reflection on Personal Experience
}

Welcome to this special issue of the International Journal of Human-Computer Studies dedicated to the innovative approaches to the development of technologies for reflection on personal experience. It is our pleasure to bring to the readers a collection of five papers selected from twenty three submissions, which bring to the foreground essential topics of this emerging research area.

This special issue follows from a workshop organised at CHI 2009 (http://www.comp.lancs.ac.uk/ corina/CHI09Workshop/) which was driven by the organisers' involvement and leadership of an ongoing initiative founded by the European Commission under Marie Curie programme: the Initial Training Network DESIRE-Creative Design for Innovation in Science and Technology (No. 215446-2).

The concept of user experience and its increasingly acknowledged role in understanding usability of technology has paved the way for experienced-based design. In this context, conceptualising the experience of interaction with technology as a reflective, sense-making endeavour has already started to emerge. Slow design, reflective design, inquisitive design, and technology as experience are attempts in this direction, which particularly highlight the role of emotions and artistic representations for inviting reflection. Although reflection is a required practice in design, we aim to broaden its scope into looking at how technology should be designed to support people's reflection, when the object of reflection is personal and directly related to everyday events in people's lives.

Designing for reflection on personal experience is an emerging field capturing the movement from designing for experience as interaction with technology towards designing for reflection on felt-life experience captured by technology. This field is positioned at the intersection of life-logging, context aware, affective, and autobiographical memory technologies. However, despite their potential, there has been surprisingly little research into how these technologies invite self reflection and support personal sense making. A key motivation of this issue is to address this gap by offering insights into the design and evaluation of technology for reflection, refinement of their design guidelines, methodological contributions to reflective design and novel theoretical perspectives informing it. 
The issue begins with an excellent paper by Thieme, Wallace, Thomas, Chen, Kraemer and Olivier who introduce Lovers' box, an innovative digital artefact supporting the exchange of video messages between romantic partners. The system was developed to support people in engaging in reflection on their intimate relationships and its evaluation involved five couples using the boxes over a period of five weeks. This paper is original in its approach to support reflection: the content to be reflected upon is not automatically captured but is created by participants themselves (with the help of a digital media artist), which in turn supports its adoption and appropriation. The paper includes insightful findings emphasising the various mirrors and sources of reflection, the design elements supporting reflection, as well as the perception of the artefact and its multiple roles in strengthening intimacy.

The second paper by Kalnikaite and Whittaker introduces MemoryLane, a digital memory application for capturing, organising and reflecting on digital representations of mementos such as pictures and audio narratives of people, places and objects. The reflection is particularly supported through annotations of mementos through speechor text-based narratives. This paper is original in its attempt to support reflection through users' activity to organise their mementos in each of the three contextual views of the MemoryLane: home, places and people. It also covers an important gap in mementos research which has focused mostly on physical objects rather than their digital representations. The system was evaluated with 31 participants who captured mementos for three consecutive days and reconstructed them through rating their importance, associated feelings and narratives.

The third paper by Lindley, Glancy, Harper, Randall and Smyth provides an in-depth analysis of how images captured by SemseCam may foster reflection on everyday experiences. The SenseCams were worn by the members of four households and reviewed one week later and 18 months later. Thus, this original paper investigates the impact on reflection on photos taken from different perspectives and on retelling their associated narratives at different moments in time. An insightful discussion highlights the features of the photos fostering reflection, together with the relevant issues of self presentation, motivation for engaging in reflection as well as the need for a dedicated time and space for engaging in reflective activities.

The fourth paper by Bowen and Petrelli offers an important methodological contribution to support reflective practice in design, and in particular the design of technology for reflection on autobiographical memories. Two field studies into families' use of physical and digital artefacts allowed the discovery and evaluation of their relevant properties to support remembering, i.e. being "not like work", discoverable and fun. Such features were further explored through three design-led workshops inspired by critical design. Its products, a range of mock-ups of critical artefacts were used both as design inspirations and sources of reflection into users' values in the context of autobiographical recollection, and the possibilities for technology application. 
The final paper by Aipperspach, Hooker and Woodruff provides design guidelines for tangible reflective technologies. It originally explores the relationship between the technology and its surrounding environment by drawing inspiration from environmental psychology theory and its concept of restorative environments. Such guidelines were applied in the design of a series of Data Souvenirs: hardware sketches combining technology with the physical form of books. The paper offers an excellent reflection on the design process based on character-based inspiration and design sketches.

We hope that the readers will enjoy this special issue and find the papers inspiring and controversial, so they indeed may foster reflection which can open avenue for further research in this emerging and exciting area.

We would like to thank the authors who helped create this special issue, and we also appreciate the collective effort of our Review Committee who has ensured the high quality of the included papers. In addition to the Guest Editors, the Review Committee consists of:

- Ann Blandford, University College London

- Ann Light, Sheffield Hallam University

- Connor Graham, University of Melbourne

- Daniela Petrelli, Sheffield Hallam University

- Deidra Morrison, Northwestern University

- Elaine Raybourn, Sandia National Laboratories

- Elise van den Hoven, Technische Universiteit Eindhoven

- Gareth Jones, Dublin City University

- Gavin Doherty, Trinity College Dublin

- Genovefa Kefalidou, Lancaster University

- Giancarlo Dimaggio, Terzo Centro di Psicoterapia Cognitiva

- Gilly Leshed, Cornell University.

- Haliyana Khalid, Lancaster University

- Helena Mentis, Microsoft Research Cambridge

- Jarmo Laaksolahti, Swedish Institute of Computer Science

- Jong Park, Korea Advanced Institute of Science and Technology

- Kristina Höök, Stockholm University

- Mark Blythe, University of York

- Matt Eliot, Central Queensland University

- Patrick Olivier, Newcastle University

- Paul Brna, University of Edinburgh

- Paula Alexandra Silva, Fraunhofer AICOS

- Richard Harper, Microsoft Research Cambridge

- Scott Mainwaring, Intel Labs

- Siân Lindley, Microsoft Research Cambridge

- Simon Bowen, Sheffield Hallam University

- Timo Partala, Tampere University of Technology

- Tom McEwan. Edinburgh Napier University

- Virpi Roto, Nokia Research Centre 
Corina $\operatorname{Sas}^{1}$

Alan Dix ${ }^{1,2}$

${ }^{1}$ School of Computing and Communications

InfoLab21, South Drive

Lancaster University

LAI 4WA, UK

Email address: corina@comp.lancs.ac.uk

${ }^{2}$ Talis

Knights Court, Solihull Parkway

Birmingham Business Park

B37 7YB, UK

Email address: alan@hcibook.com 